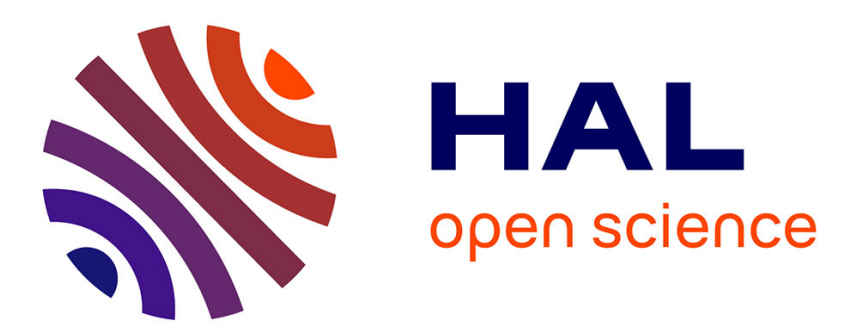

\title{
Tuning Memristivity by Varying the Oxygen Content in a Mixed Ionic-Electronic Conductor
}

Klaasjan Maas, Edouard Villepreux, David Cooper, Eduardo Salas-Colera, Juan Rubio- Zuazo, German R Castro, Olivier Renault, Carmen Jiménez, Herve Roussel, Xavier Mescot, et al.

\section{To cite this version:}

Klaasjan Maas, Edouard Villepreux, David Cooper, Eduardo Salas-Colera, Juan Rubio- Zuazo, et al.. Tuning Memristivity by Varying the Oxygen Content in a Mixed Ionic-Electronic Conductor. Advanced Functional Materials, 2020, 30 (17), pp.1909942. 10.1002/adfm.201909942 . hal-02994852

\section{HAL Id: hal-02994852 \\ https://hal.science/hal-02994852}

Submitted on 10 Jun 2021

HAL is a multi-disciplinary open access archive for the deposit and dissemination of scientific research documents, whether they are published or not. The documents may come from teaching and research institutions in France or abroad, or from public or private research centers.
L'archive ouverte pluridisciplinaire $\mathbf{H A L}$, est destinée au dépôt et à la diffusion de documents scientifiques de niveau recherche, publiés ou non, émanant des établissements d'enseignement et de recherche français ou étrangers, des laboratoires publics ou privés. 
Tuning memristivity by varying the oxygen content in a mixed ionic-electronic conductor

Klaasjan Maas, Edouard Villepreux, David Cooper, Eduardo Salas- Colera, Juan RubioZuazo, German R. Castro, Olivier Renault, Carmen Jimenez, Hervé Roussel, Xavier Mescot, Quentin Rafhay, Michel Boudard, Mónica Burriel*

Dr. K. Maas, Dr. C. Jimenez, Dr. H. Roussel, Dr. M. Boudard, Dr. M. Burriel, Univ. Grenoble Alpes, CNRS, Grenoble INP ${ }^{\dagger}$, LMGP, F-38000 Grenoble, France

${ }^{\dagger}$ Institute of Engineering Univ. Grenoble Alpes

*E-mail: monica.burriel@grenoble-inp.fr

Dr. Q. Rafhay, Dr. X. Mescot

Univ. Grenoble Alpes, CNRS, IMEP-LAHC, F-38000 Grenoble, France

E. Villepreux, Dr. D. Cooper, Dr. O. Renault

Univ. Grenoble Alpes, CEA, LETI, 38000 Grenoble, France

Dr. E. Salas- Colera, Dr. G. R. Castro, Dr. J. Rubio-Zuazo

Spanish CRG BM25-SpLine Beamline at the ESRF, 71 avenue des Martyrs 38000 Grenoble, France and Instituto de Ciencia de Materiales de Madrid-ICMM/CSIC, Cantoblanco, E-28049 Madrid, Spain

Keywords: Mixed ionic electronic conductors, memristive devices, valence change memories, $\mathrm{La}_{2} \mathrm{NiO}_{4}$, neuromorphic

\footnotetext{
Abstract: The rising interest shown for adaptable electronics and brain inspired neuromorphic hardware opens the way to new device architectures and functional materials being used to build these devices. The rational design of these memory components also 1
} 
benefits the comprehension and thus the control over the microscopic mechanisms at the origin of memristivity. In oxide-based Valence-Change Memories (VCMs) the control of the oxygen drift and diffusion kinetics is a key aspect in obtaining the gradual analog-type change in resistance required for artificial synapse applications. However, only a few devices have been designed with this in mind, as they are commonly built around ionic insulating active materials. Here this shortcoming is addressed by using a Mixed Ionic-Electronic Conductor (MIEC) as functional memristive material. This work demonstrates how the oxygen content in $\mathrm{La}_{2} \mathrm{NiO}_{4+\delta}$ (L2NO4), tuned through post-annealing treatments, has a critical influence on the memory characteristics of L2NO4-based memristive devices. The presence of interstitial oxygen point defects in L2NO4 affects both its structure and electrical properties. High oxygen stoichiometry in the pristine state leads to an increased electrical conductivity, ultimately resulting in an improved memory window with highly multilevel, analog-type memory programing capabilities, desirable for analog computing and synaptic applications in particular.

\section{Introduction.}

Valence-Change Memories (VCMs) are a type of bipolar memristive devices in which internal redox reactions govern the resistance-change mechanism. ${ }^{[1]}$ More particularly, in oxides, the motion of oxygen ions is held responsible for the local valence-change (redox reaction) in the cation sublattice, ${ }^{[1]}$ resulting in a change in the electronic conductivity, which magnitude depends on the structure of the device and its constituent materials. VCMs are one of the most promising technologies for the hardware implementation of neuromorphic computing, as they combine a large number of desired properties including speed, scalability, low power consumption and CMOS compatibility ${ }^{[2]}$. In addition, under certain operating conditions VCMs can present analog conductance (resistance) modulation, a critical 
requirement for memristors when used for computing. Significant examples of the use of memristors for neuromorphic computing include the simulation of both neuronal and synaptic dynamics including spike-timing-dependent plasticity (STDP) ${ }^{[2]}$ and the full hardwareimplementation of convolutional neural networks (CNNs) for image recognition using memristor crossbars. ${ }^{[2]}$

The VCM mechanism can exhibit both filamentary and interface-type switching, classified as such depending on whether the region of the device responsible for the change in resistance is localized (filamentary) or widespread across the electrode/oxide interface (i.e. interfacetype). ${ }^{[3]}$ Despite filamentary switching being the most commonly used type of VCM, there is currently little control over the formation and properties of the conducting filament, which creation usually requires an electroforming. This destructive biasing step is necessary to give rise to the memory properties and can be understood as a soft dielectric breakdown of the sandwiched material leading to the stochastic creation of filamentary pathways which can connect and disconnect the two electrodes. Conversely, in interface-type VCMs, the resistance-change is usually attributed to a field-induced change of a Schottky barrier at the electrode/oxide heterojunction. ${ }^{[1]}$ The externally-applied electric field induces a drift of oxygen ions which accumulate at the positive electrode, locally changing the junction properties, such as the depletion width of the majority carriers. Besides changing the contact characteristics, the accumulation of oxygen ions near the electrode can also lead to the generation of oxide interlayers. This process mostly depends on the Gibbs free energy for the formation of the metal-oxide phase (from the electrode material). The additional oxide interlayer then adds a series resistance to the device, increasing its overall resistance and can trigger a memristive behavior.

Some materials are more prone than others to result in interface-type VCM. In particular, we have used the following criteria to select $\mathrm{La}_{2} \mathrm{NiO}_{4+\delta}$ as valence change oxide for the design of 3 
our interface-type VCM,: 1) Oxygen storage: stable material (it should not decompose) when varying its oxygen stoichiometry; 2) Oxygen transport: the material should be able to supply oxygen towards the metal/oxide interface in its bulk, allowing for an homogeneous change in resistance to occur across the entire metal/oxide interface; and 3) Electronic conduction: the material should not be an insulator to prevent dielectric breakdown (in order to avoid filamentary-type switching). In order to extend the range of materials showing interface-type memristance and to adapt the choice to the targeted application and time-response required, these three criteria can applied in the future as selection rules in the search for new memristive oxides.

\section{1. $\mathrm{La}_{2} \mathrm{NiO}_{4+\delta}$, a layered structure with mixed conduction properties.}

$\mathrm{La}_{2} \mathrm{NiO}_{4+\delta}$ (L2NO4) has been selected for the design of interface-type VCMs due to its oxygen storage, oxygen transport and electronic conduction properties. L2NO4 is a Mixed Ionic-Electronic Conductor (MIEC), combining good electronic conductivity, with high oxygen exchange and diffusion coefficients. ${ }^{[4]}$ It has a rich phase diagram which allows it to oscillate between two main crystal structures being: I4/mmm, a high symmetry tetragonal phase and Bmab, a lower symmetry orthorhombic phase. This phase transition is both temperature and $\mathrm{pO}_{2}$-dependent. ${ }^{[5]}$ L2NO4 also has the particularity of accommodating interstitial oxygen anions within its lattice. This additional oxygen is key to the bulk oxygen

conduction exhibited by the material. ${ }^{[6]}$ A large range of oxygen over-stoichiometry can be stabilized in L2NO4 without it decomposing, ${ }^{[7]}$ making it a potentially good oxygen storage medium. The high symmetry tetragonal structure of L2NO4 as well as the location of the interstitial oxygen and the naming convention used throughout the text for the different atoms and bonds composing the material are shown in Figure 1a and b. Concerning its bulk oxygen-ion diffusion capabilities, both experimental and computational evidence has shown that an insterstitialcy diffusion mechanism is the preferred pathway for a fast diffusion along 4 
the $a b$ plane (around three orders of magnitude higher than along the $c$ axis). ${ }^{[4,8-10]}$ There is an agreement between the measured and the calculated activation energy for this diffusion process $\left(\mathrm{E}_{\mathrm{a}} \approx 0.88 \mathrm{eV}\right)^{[8,11,12]}$, which is significantly lower than for other well-known ionic conductors (e.g yttria-stabilized-zirconia has an activation energy of $1 \mathrm{eV}^{[13]}$ ). This low value together with a very high diffusion coefficient is even more relevant when compared to other oxides used as memristive materials which are not good ionic conductors in their pristine state (eg: $\mathrm{SrTiO}_{3}, \mathrm{Al}_{2} \mathrm{O}_{3}, \mathrm{Ta}_{2} \mathrm{O}_{5}, \mathrm{HfO}_{2}$, see ref. ${ }^{[14]}$ for more details). However, it is possible that ionic insulators might in fact show significant oxygen diffusion capabilities after being electroformed or if they contain a high density of defects introduced (intentionally or not) during processing.

(a)

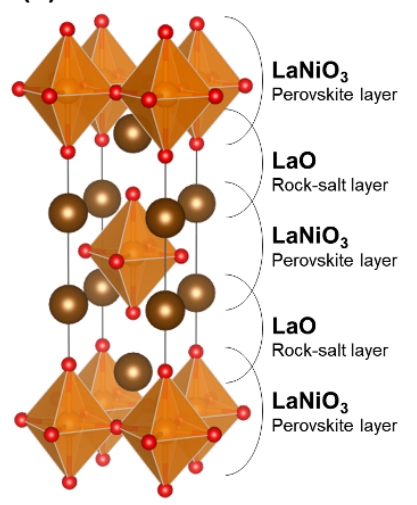

(b)

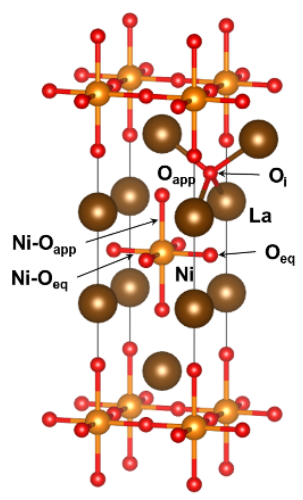

(c)

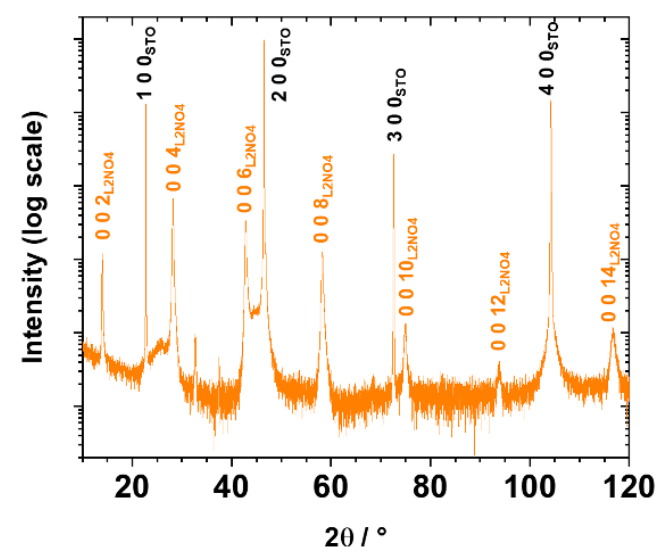

Figure 1. (a) Schematic representation of the tetragonal structure of stoichiometric $\mathrm{La}_{2} \mathrm{NiO}_{4.00}$ showing the alternating Perovskite and rock-salt type layers, which is at the origin of the anisotropic conduction properties of the material. (b) Naming convention used in the text for the different atoms and their bounds composing the structure. $O_{\text {app }}$ stands for "apical oxygen atom", $O_{\text {eq }}$ for "equatorial oxygen atom", $O_{i}$ for "interstitial oxygen atom", $\mathrm{Ni}-\mathrm{O}_{\text {app }}$ for "nickel-apical oxygen bond" and $\mathrm{Ni}-\mathrm{O}_{\mathrm{eq}}$ for "nickel-equatorial 
oxygen bond". L2NO4 is represented in the space group I4/mmm (SG $\mathrm{n}^{\circ} 139$ ). (c) Out of plane XRD pattern of a L2NO4/STO sample (acquired before deposition of the top electrodes).

The presence of oxygen interstitials $\left(O_{i}^{\prime \prime}\right.$, using the Kroger-Vink defect notation) has to be electrically compensated by positive holes to keep the overall charge-neutrality within the L2NO4 lattice. This charge compensation takes place according to the following equation:

$$
\frac{1}{2} O_{2, \text { gas }}+V_{i}^{x}=O_{i}^{\prime \prime}+2 h^{\bullet}
$$

where $O_{2 \text {,gas }}$ refers to molecular oxygen present in the gas phase and where the other defect elements are described by Kröger-Vink notation: $V_{i}^{x}$ is a neutral vacant interstitial site, $O_{i}^{\prime \prime}$ denotes a doubly negatively-charged oxygen ion in an interstitial site and $h^{\bullet}$ represents a positively-charged hole carrier. Equation $1 \frac{1}{2} O_{2, \text { gas }}+V_{i}^{x}=O_{i}^{\prime \prime}+2 h^{\bullet} \frac{1}{2} O_{2, \text { gas }}+V_{i}^{x}=O_{i}^{\prime \prime}+$ $\mathbf{2 h}^{\bullet}$ shows that the amount of $O_{i}^{\prime \prime}$ present in L2NO4 is directly correlated to the hole carrier concentration. It is in this sense that $O_{i}^{\prime \prime}$ can play the role of an acceptor dopant, giving rise to the p-type conductivity reported for this material. ${ }^{[15-17]}$ The large decrease in resistivity with increasing oxygen content is clearly demonstrated by Thanh et al., ${ }^{[18]}$ who had also been the sole to report on the resistive switching characteristics of L2NO4-based devices. In this work they used bulk L2NO4 samples with Ag symmetric electrodes and showed a certain influence of the oxygen content in the resistance switching ratio.

Aiming to the real implementation and functional optimization of this MIEC oxide in memristive devices we recently reported for the first time on the switching characteristics of L2NO4 thin film (67 $\mathrm{nm}$ ) devices with $\mathrm{Pt}$ and $\mathrm{Ti}$ as inert and active electrodes, respectively. The n-type $\mathrm{TiO}_{\mathrm{x}}$ interface layer that is naturally formed at the $\mathrm{Ti} / \mathrm{L} 2 \mathrm{NO} 4$ interface gives rise to remarkable analog switching capabilities, presenting very gradual set and reset transitions, optimal for the hardware implementation of synaptic functions. ${ }^{[14]}$ However, the role of the 
interstitial oxygen ions (and concomitant presence of positively-charged hole carrier) in the memristive behavior of these novel VCM devices had not yet been studied. We use a planar, or "top-top" electrode configuration with macroscopic distances (a few hundreds of microns) between the electrodes to avoid the formation of conductive filaments and to have a good control over the interface-type resistance changes in the memristive devices. In the conventional top-bottom "sandwich-type" configuration the low series resistance of L2NO4 (tens of nm-thick) is expected to lead to high currents, subsequent Joule heating and can ultimately induce an important degradation of the electrode material and/or the sandwiched material. While $\mathrm{Cu}$ or Ag-based Electro-Chemical Metallization (ECM) cells have been largely studied for their filamentary resistive switching mechanism, the formation of $\mathrm{Pt}$ filament in a $\mathrm{Pt} / \mathrm{TiO}_{2} / \mathrm{Pt}$ memristive device has also been reported, ${ }^{[19]}$ suggesting that the majority of electrode materials can migrate through the cell and short circuit the device if exposed to harsh programming conditions.

In this paper, we studied the effect of varying the oxygen content of the L2NO4 mixed conductor on the electrical and memristive properties of planar Pt/L2NO4/Ti thin film devices. High oxygen contents in the pristine state enhance the memory properties of these devices by offering increased multilevel capabilities as well as a larger programming window.

\section{Results and Discussion}

\subsection{Deposition of L2NO4 thin films and fabrication of Pt/L2NO4/Ti devices.}

The optimal growth conditions for L2NO4 thin films are summarized in Table 1. Using these conditions, it was possible to grow highly oriented films on $\left[\begin{array}{lll}1 & 0 & 0\end{array}\right]$-oriented $\mathrm{SrTiO}_{3}$ (STO) single crystal substrates.

Table 1: Optimized deposition conditions used for the growth of L2NO4 thin films by PiMOCVD.

Parameter Value 


\begin{tabular}{ll}
\hline Precursors & $\mathrm{La}(\mathrm{TMHD})_{3}$ and Ni(TMHD $)_{2}$ \\
Solution concentration & $0.02 \mathrm{~mol} / \mathrm{L}$ (solvent $=\mathrm{m}$-xylene) \\
Injection frequency & $1 \mathrm{~Hz}$ \\
Opening time & $2 \mathrm{~ms}$ \\
Number of injected droplets & 4000 \\
Evaporation temperature & $220{ }^{\circ} \mathrm{C}-280{ }^{\circ} \mathrm{C}($ several heating stages $)$ \\
Substrate temperature & $650{ }^{\circ} \mathrm{C}$ \\
Carrier gas & $34 \% \mathrm{Ar}(218 \mathrm{sccm})+66 \% \mathrm{O}_{2}(418 \mathrm{sccm})$ \\
Total pressure inside the reactor & 5 Torr
\end{tabular}

The crystal quality and preferential orientation of the L2NO4 films can be observed in Figure 1.d where the $\theta-2 \theta$ X-ray diffraction (XRD) patterns show only the $001_{\mathrm{L} 2 \mathrm{NO} 4}$ (1 even) reflections of the $\mathrm{L} 2 \mathrm{NO} 4$ phase in addition to the $\mathrm{h} 00_{\text {STO }}$ substrate peaks.

\subsection{Tuning the oxygen content in L2NO4 through post annealing treatments.}

In order to evaluate how the oxygen stoichiometry of L2NO4 affects its conduction and memristance properties, a batch of four L2NO4/STO samples was deposited simultaneously in the PiMOCVD reactor. One of the samples was kept as deposited (pristine) while the other three were annealed in $\mathrm{O}_{2}$, Ar or a highly reducing $\mathrm{H}_{2}(6 \%) / \mathrm{Ar}$ gas mixture at $500{ }^{\circ} \mathrm{C}$ for $1 \mathrm{~h}$ prior to top electrode deposition. The diffractogram in Figure 2.a shows a clear displacement of the $006_{\mathrm{L} 2 \mathrm{NO} 4}$ peak to higher $2 \theta$ angles (lower interplanar spacing along the $c$ crystallographic direction of L2NO4) when the samples are subjected to reducing conditions. This is in agreement with a loss of oxygen, and more particularly of interstitial oxygen. ${ }^{[7]}$ The Ni oxidation state was determined from X-ray Absorption Near-Edge Structure (XANES) experiments by measuring the displacement of the Ni-K edge, from which the $\delta$ value could be estimated. The inset in Figure 2.b shows the shift of the Ni K-edge towards higher 8 
energies when the samples are treated in increasingly oxidizing atmospheres, from which the $\mathrm{Ni}$ oxidation state was calculated using as references the $\mathrm{Ni}-\mathrm{K}$ edge positions measured by Woolley et al. (see supplementary information (SI), Figure S3, for more details). ${ }^{[20]}$ The oxidation state of Ni then allows the calculation of the oxygen off-stoichiometry by chargebalance. The results are shown in Table 2 where they are compared to the values obtained by Woolley et al. who used the same technique to determine the $\delta$ value of L2NO4 ceramic samples under different measurement conditions. ${ }^{[20]}$

Table 2: Measured Ni K-edge position (XANES) and corresponding values of the Ni oxidation state and oxygen over-stoichiometry $\delta$ in L2NO4.

\begin{tabular}{|c|c|c|c|c|c|c|c|}
\hline & Material & $\begin{array}{l}\text { Annealing } \\
\text { conditions }\end{array}$ & $\begin{array}{c}\text { Measurement } \\
\text { conditions }\end{array}$ & $\begin{array}{c}\text { Ni K-edge } \\
\text { position } \\
\text { (eV) }\end{array}$ & $\begin{array}{c}\mathrm{Ni} \\
\text { oxidation } \\
\text { state }\end{array}$ & $\begin{array}{l}\text { estimated } \\
\delta \text { value }\end{array}$ & Reference \\
\hline \multirow{3}{*}{$\begin{array}{c}\text { Calibration } \\
\text { data }\end{array}$} & Ni foil & - & RT in air & 8333.0 & 0 & - & This work \\
\hline & $\mathrm{NiO}$ & - & RT in air & 8345.2 & 2 & - & {$[20]$} \\
\hline & $\mathrm{LaNiO}_{3}$ & - & RT in air & 8348.2 & 3 & - & [20] \\
\hline \multirow{3}{*}{$\begin{array}{l}\text { Ceramic } \\
\text { samples }\end{array}$} & $\mathrm{La}_{2} \mathrm{NiO}_{4+\delta}$ & & RT in air & 8346.1 & 2.24 & 0.12 & [20] \\
\hline & $\mathrm{La}_{2} \mathrm{NiO}_{4+\delta}$ & & at $650^{\circ} \mathrm{C}$ in air & 8345.5 & 2.15 & 0.08 & [20] \\
\hline & $\mathrm{La}_{2} \mathrm{NiO}_{4+\delta}$ & & at $650^{\circ} \mathrm{C}$ in $\mathrm{N}_{2}$ & 8347.2 & 2.08 & 0.04 & [20] \\
\hline \multirow{4}{*}{ Thin films } & $\mathrm{La}_{2} \mathrm{NiO}_{4+\delta}$ & $\begin{array}{l}\mathrm{O}_{2}, 500{ }^{\circ} \mathrm{C} \\
\text { during } 1 \mathrm{~h}\end{array}$ & RT in air & 8345.76 & 2.17 & 0.09 & This work \\
\hline & $\mathrm{La}_{2} \mathrm{NiO}_{4+\delta}$ & pristine & RT in air & 8345.54 & 2.11 & 0.05 & This work \\
\hline & $\mathrm{La}_{2} \mathrm{NiO}_{4+\delta}$ & $\begin{array}{l}\text { Ar, } 500{ }^{\circ} \mathrm{C} \\
\text { during } 1 \mathrm{~h}\end{array}$ & RT in air & 8345.47 & 2.08 & 0.04 & This work \\
\hline & $\mathrm{La}_{2} \mathrm{NiO}_{4+\delta}$ & $\begin{array}{l}\mathrm{H}_{2}, 500{ }^{\circ} \mathrm{C} \\
\text { during } 1 \mathrm{~h} \\
\end{array}$ & RT in air & 8345.27 & 2.02 & 0.01 & This work \\
\hline
\end{tabular}

The $c$ lattice expansion was calculated form the $006_{\mathrm{L} 2 \mathrm{NO} 4}$ peak position and is plotted as a function of the oxygen off-stoichiometry $\delta$ in Figure 2.c. A linear relationship is observed between the $c$ lattice parameter and the oxygen off-stoichiometry (black line). The out of plane lattice parameter increases from $c=12.62 \AA$ to $12.69 \AA$ when the estimated oxygen over-stoichiometry varies from $\delta=0.01$ to 0.09 . Although L2NO4 is expected decompose 
into $\mathrm{La}_{2} \mathrm{O}_{3}+\mathrm{Ni}$ at very high temperatures and under very reducing conditions (for example $\mathrm{T}$ $=1373 \mathrm{~K}$ and $\mathrm{pO}_{2}<10^{-10}$ bar), ${ }^{[21]}$ no significant difference was observed when comparing the integrated intensity of the diffraction peaks relative to the L2NO4 phase before and after annealing at this relatively low temperature and time $(727 \mathrm{~K}, 1 \mathrm{~h})$. We can thus conclude that the post-annealing treatments did not lead to a degradation of the L2NO4 film, as also confirmed by the similar morphologies of the SEM images taken of the surface of each sample (displayed in Erreur! Source du renvoi introuvable..a-d, SI) and by the fact that no additional peaks were observed by XRD after the treatments. 
(a)

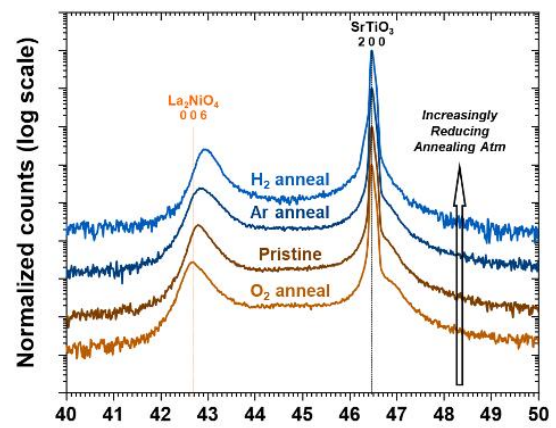

(b)

$2 \theta / \circ$

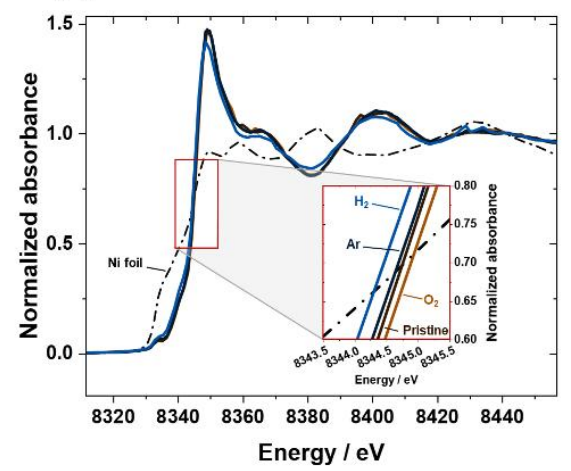

(c)

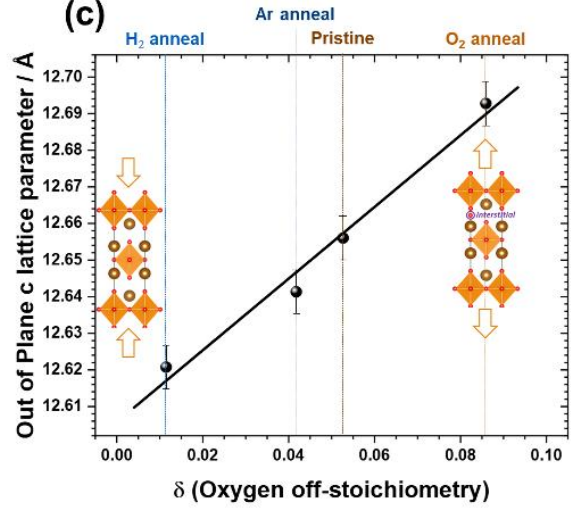

Figure 2. (a) Out of plane $\theta$-20 XRD pattern of L2NO4/STO samples with different gas atmosphere anneals $\left(500{ }^{\circ} \mathrm{C} / 1 \mathrm{~h}\right)$. A clear displacement of the out of plane $006_{\mathrm{L} 2 \mathrm{NO} 4}$ Bragg peak to higher $2 \theta$ values (lower interplanar spacing) is observed. The out-of-plane $c$ parameter equals $12.62 \AA, 12.64 \AA, 12.66 \AA$ and $12.69 \AA$ for the $\mathrm{H}_{2}$, Ar, pristine and $\mathrm{O}_{2}$ anneal, respectively. This behavior is expected when interstitial oxygen ions are removed/introduced from/in the lattice. (b) XANES spectra at the Ni K-edge obtained for L2NO4 with different gas anneals. The inset shows a magnification of the edge area, highlighting the shift of the $\mathrm{Ni}$ K-edge towards higher energies when the samples are treated in increasingly oxidizing atmospheres. A solid Ni foil was used as reference for the calibration of the curves (dashed-dotted line). The edge position was obtained using the first/second derivative method. (c) 11 
Evolution of the $c$ lattice parameter and film resistivity of L2NO4 with oxygen off-stoichiometry $\delta$ ( $\delta$ equals $0.01,0.04,0.05$ and 0.09 , respectively). The $\delta$ value was estimated the displacement of the Ni K-edge measured by XANES (as shown in (b)). All the films are $\sim 67 \mathrm{~nm}$ thick.

\subsection{The presence of a $\mathrm{TiO}_{\mathrm{x}}$ functional interlayer.}

Pt and Ti electrodes (200 nm thick) were evaporated on top of the L2NO4 thin film to construct the memristive devices. When two inert Pt electrodes were used to construct $\mathrm{Pt} / \mathrm{L} 2 \mathrm{NO} 4 / \mathrm{Pt}$ devices no memristivity was obtained as, as shown in Figure S2, regardless of the post annealing treatment $\left(\mathrm{O}_{2}\right.$, Ar or $\left.\mathrm{H}_{2}\right)$ the electrical characteristics remain completely ohmic up to $\pm 10 \mathrm{~V}$. Although Pt forms an ohmic contact with L2NO4, ${ }^{[14]}$ Ti tends to naturally form an oxide (i.e. $\mathrm{TiO}_{\mathrm{x}}$ ) interlayer when put in contact with an oxide. ${ }^{[14,22-25]}$ The presence and composition of this interlayer in the Ti/L2NO4/STO stack was analyzed in detail. Figure 3.a shows the atomic composition maps (for $\mathrm{Ti}, \mathrm{O}, \mathrm{Ni}$ and $\mathrm{La}$ ) obtained by Electron-Energy Loss Spectroscopy (EELS) on a TEM cross-section specimen taken beneath a Ti top electrode. The L2NO4 film appears continuous, dense and homogeneous in composition (no visible gradient in the $\mathrm{O}, \mathrm{Ni}$ or La maps), with a thickness of $\sim 67 \mathrm{~nm}$. It can be noticed that an additional inhomogeneous darker contrast is present between the L2NO4 thin film and the Ti top electrode in the Annular Dark Field (ADF) image shown at the top of Figure 3.a. We have previously shown that this interlayer corresponds to a sub-stoichiometric $\mathrm{TiO}_{\mathrm{x}}$ interlayer which grows spontaneously during the evaporation of $\mathrm{Ti}$ through the electrode microfabrication process. ${ }^{[14]}$ The uniformity, thickness and chemical nature of this interlayer was analyzed in detail by Electron Energy Loss Spectrum (EELS) using an advanced spectral unmixing technique (known as the Vertex Component Analysis method, or VCA) and focusing in particular on the convoluted O K-edge Energy Loss Spectrum (ELS) (more details about this technique are available in the SI). The optimized results for the spectral unmixing were obtained using four components, corresponding to the four different phases appearing in 12 
the EELS maps: one for the STO substrate, one for the L2NO4 oxide thin film, one for the $\mathrm{TiO}_{\mathrm{x}}$ interlayer and finally one for the Ti top electrode. The resulting $\mathrm{C} 1, \mathrm{C} 2, \mathrm{C} 3$ and $\mathrm{C} 4$ abundance maps and their corresponding spectral components are displayed in Figure 3.b and c, respectively. First, it can be noticed that the calculated ELS corresponding to $\mathrm{C} 1$ is remarkably similar to the experimental spectrum measured in epitaxial thin films of defectfree STO, ${ }^{[26]}$ and as expected, the location where this component is most present is in the STO substrate region (Figure 3.b). The second spectral component (C2) does not contain an oxygen fingerprint, it is a pure background signal which implies that there is no oxygen present in the upper part of the Ti electrode. As the corresponding abundance map suggests, component C3 corresponds to the fingerprint of oxygen in a L2NO4 environment, in agreement with the spectrum reported by Ravkina et al. ${ }^{[27]}$ Finally, the last spectrum $(\mathrm{C} 4)$ confirms the presence of oxygen chemically bounded to $\mathrm{Ti}$ in the interfacial region of the sample, forming an amorphous $\mathrm{TiO}_{\mathrm{x}}$ layer with a shape very similar to the reference spectrum for amorphous $\mathrm{TiO}_{2}$ reported in ${ }^{[28]}$. 
(a)

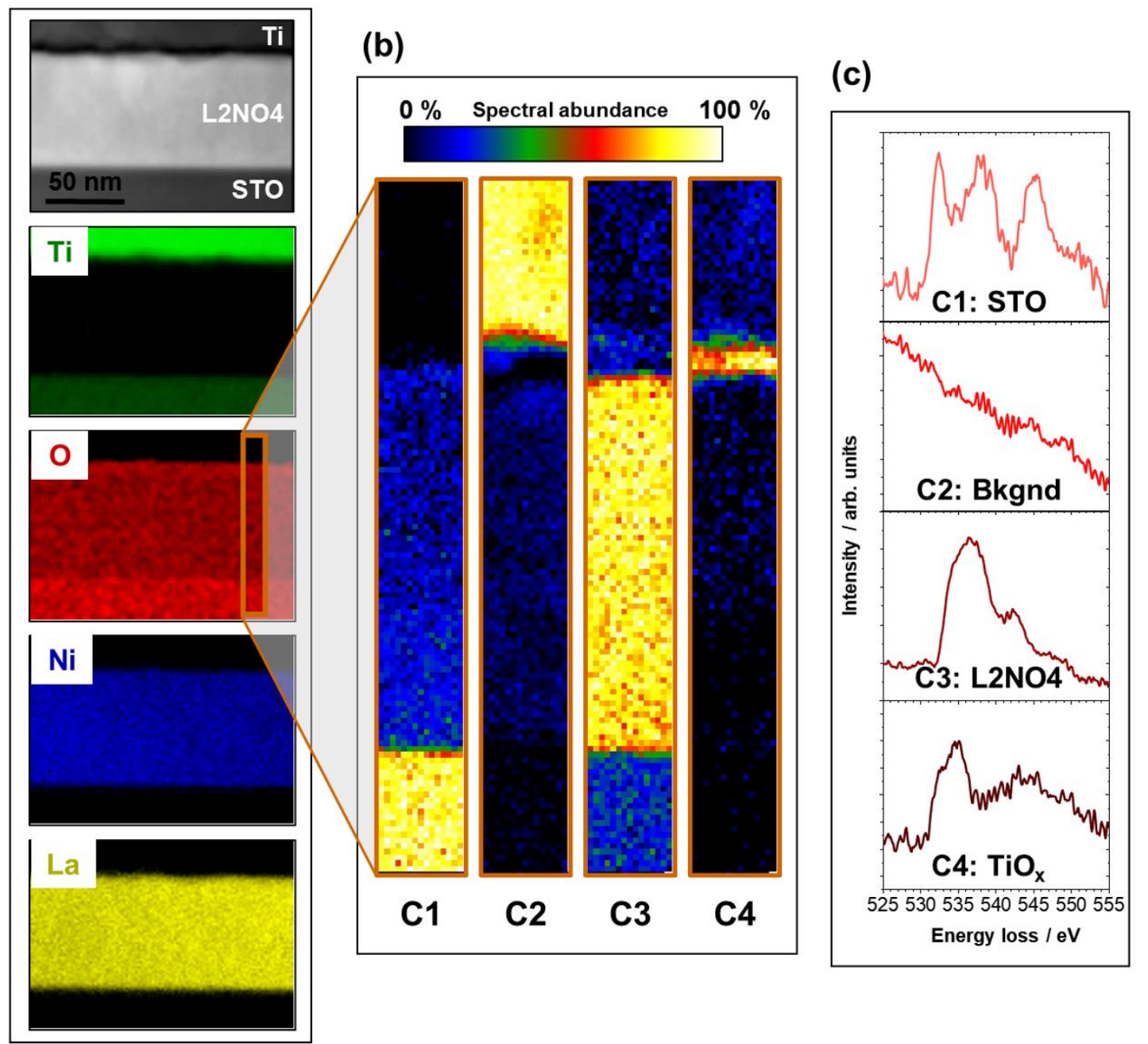

Figure 3: (a) EELS composition maps (in atoms per unit volume) for Ti, O, Ni and La taken on a Ti/L2NO4/STO TEM cross-section specimen. The O-K ( $532 \mathrm{eV})$, Ti-L2 ( 462 eV) and L3 ( 456 eV), NiL2 $(\sim 872 \mathrm{eV})$ and L3( $(855 \mathrm{eV})$ La-M4 $(\sim 849 \mathrm{eV})$ and M5 $(\sim 832 \mathrm{eV})$ edges were used for quantification. The top image corresponds to a wide angle ADF image of the entire stack showing the region of the sample used for the mapping. (b) Heat maps showing the fractional abundance of the 4 components displayed in (c) which describe the different oxygen environments present in the stack. (c) The hyperspectral O K-edge measured by EELS in each pixel of the image was unmixed using a VCA data processing technique. The resulting component spectra $\mathrm{C} 1, \mathrm{C} 2, \mathrm{C} 3$ and $\mathrm{C} 4$ are fingerprints of oxygen in a

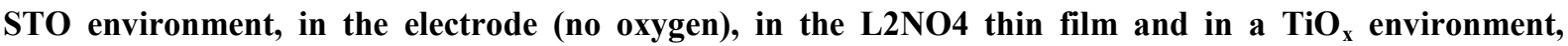
respectively. 


\subsection{Role of the oxygen content in the resistivity of the L2NO4 thin films.}

The resistivity values of the different films plotted in Figure 4 show a steady increase in resistivity from $5.3 \times 10^{-3} \Omega . \mathrm{cm}$ to $1.3 \times 10^{-2} \Omega . \mathrm{cm}$ when decreasing the $\delta$ oxygen content from 0.09 to 0.04 . Interestingly, the film annealed in a highly reducing $\mathrm{H}_{2} / \mathrm{Ar}$ atmosphere corresponds to close to stoichiometric L2NO4 (estimated $\delta=0.01$ ) and shows an increase in resistivity of over two orders of magnitude to $5.7 \Omega$.cm. Similar values of resistivity $(\sim 5 \Omega . \mathrm{cm}$ at $300 \mathrm{~K}$ ) have been reported for samples annealed in a highly reducing $\mathrm{CO} / \mathrm{CO}_{2}$ atmosphere. ${ }^{[2]}$ These results confirm that the oxygen content has an important influence on the electrical properties of $\mathrm{L} 2 \mathrm{NO} 4$ and that changes in the measured film resistivity can be directly related to the changes in the $\mathrm{Ni}$ oxidation state, oxygen content and $c$ lattice parameter in the L2NO4/STO thin films (shown in Figure 2.c).

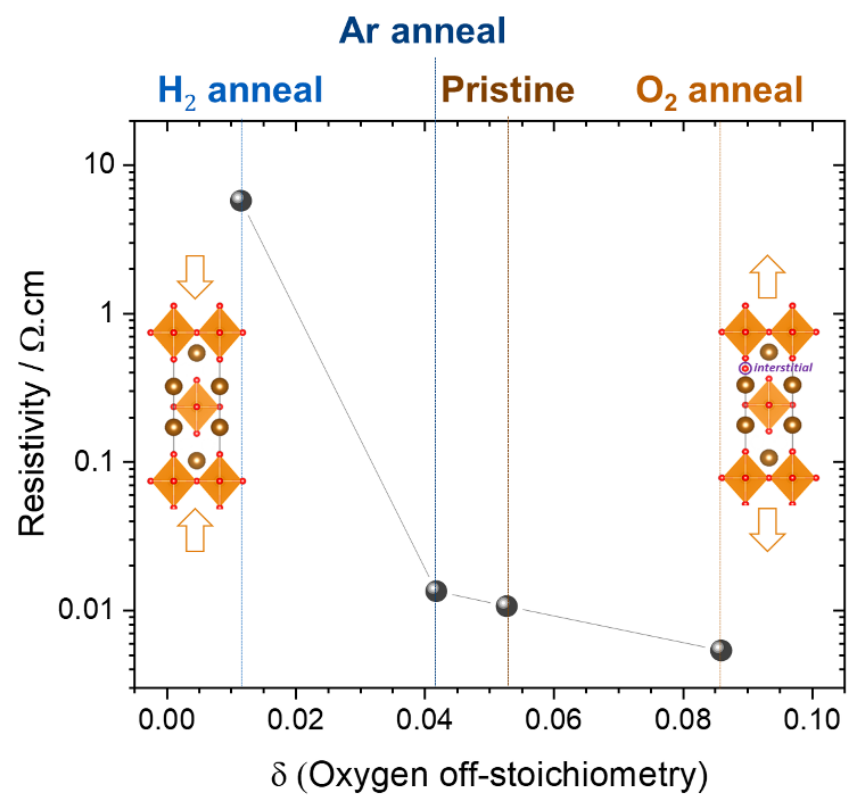

Figure 4: Evolution of the L2NO4 film resistivity with increasing oxygen stoichiometry ( $\delta$ equals 0.01 , $0.04,0.05$ and 0.09 , respectively).

Photoemission Electron Microscopy (PEEM) measurements confirmed the p-type semiconducting properties of L2NO4. The work function of two different L2NO4 films, one 15 
pristine and one annealed in oxygen, were measured using PEEM by determining the onset of the secondary electron emission spectrum. The oxidizing treatment lead to an increase of 200 $\mathrm{meV}$ in the work function of $\mathrm{L} 2 \mathrm{NO} 4$ (from $4.1 \mathrm{eV}$ to $4.3 \mathrm{eV}$ for the pristine and the oxygenannealed films, respectively). This is consistent with the interstitial oxygen ions acting as acceptor dopants through a self-doping process, as previously described, assuming however that the presence of the interstitial point defect has no significant influence in the band gap of L2NO4.

\subsection{Role of the oxygen content on the memristive properties of Pt/L2NO4/Ti devices.}

To construct the L2NO4-based memristive devices Pt has been used as chemically inactive ohmic contact, so that the Pt/L2NO4 junction offers little resistance to the passage of current. Conversely, titanium was selected for its high oxygen affinity, making it a chemically active electrode that can easily form a $\mathrm{TiO}_{\mathrm{x}}$ interlayer at the $\mathrm{Ti} / \mathrm{L} 2 \mathrm{NO} 4$ junction as shown in Figure 3. This oxygen deficient interlayer may then play the role of an oxygen storage/release medium. The exchange of oxygen between the two materials by reversible redox reactions provides an effective playground for VCMs. A sketch of the Pt/L2NO4/Ti device is presented in Figure 5.a.

To illustrate the key role of oxygen on the memristive characteristics, three Pt/L2NO4/Ti devices with increasing oxygen content in L2NO4 were programmed from their Initial Resistance State (IRS) to a High Resistance State (HRS) using the standardized initialization procedure which is described in the Experimental Section (and illustrated at the bottom of Figure 5.c). The results for the three $\mathrm{Pt} / \mathrm{L} 2 \mathrm{NO} 4 / \mathrm{Ti}$ samples with different oxygen contents, all presenting a gradual initialization behavior (no abrupt change in resistance), are displayed in Figure 5.c. The IRS, stable during the first 100 s-long readout, is different for the three samples being, as expected, larger for the samples prepared in reducing conditions $\left(4.6 .10^{5} \Omega\right.$, 4.4.10 $\Omega$ and $2.2 .10^{3} \Omega$ for the hydrogen, argon and oxygen-annealing, respectively). When 
applying the train of write pulses, the samples follow two different behaviors depending on if they had been previously reduced or oxidized. The resistance of the two reduced samples decreases gradually during the first ten write pulses before increasing and retrieving the IRS after 55 and 42 pulses for the hydrogen and argon-annealed samples, respectively. Interestingly, the oxygen annealed sample does not show the initial decrease in resistance and is directly programmed to a higher resistance state showing first a slow increase in resistance (during the first 40 pulses, approx. 500s) followed by a steeper increase. The initial resistance drop for the two reduced samples could originate from the field-induced drift of the interstitials $\left(O_{i}^{\prime \prime}\right)$ present in the sample towards the Ti/L2NO4 interface. This would reduce the $\mathrm{Ti} / \mathrm{L} 2 \mathrm{NO} 4$ contact resistance $\left(\mathrm{R}_{\mathrm{C}, \mathrm{Ti}}\right)$ due to a local dopant increase at the Ti/L2NO4 interface, hence decreasing the carrier depletion width at this location (schematic illustrations depicting the proposed mechanisms shown in Figure S4). At a certain threshold concentration of $O_{i}^{\prime \prime}$ (achieved after the first ten write pulses), the oxygen ions could start drifting into the Ti electrode and increase the resistance of the device by increasing the $\mathrm{TiO}_{\mathrm{x}}$ interlayer thickness and/or its oxygen stoichiometry (making it more insulating). In the case of the oxygenannealed sample, the oxygen concentration at the Ti/L2NO4 interface should already be high initially, so that oxygen ions can start drifting into the Ti electrode and oxidizing it as soon as the first write pulse is applied.

At the end of the initialization procedure, the resistance of the three samples continues to increase spontaneously (i.e. without the application of an external electric field) during the $100 \mathrm{~s}$ long readout step. This effect could be explained by the diffusion of the oxygen ions away from the $\mathrm{Ti} / \mathrm{L} 2 \mathrm{NO} 4$ interface to restore a homogeneous chemical gradient across the L2NO4 film and/or a stable space-charge zone near the Ti/L2NO4 interface (see Figure S4). Although a certain $\mathrm{TiO}_{\mathrm{x}}$ formation cannot be excluded and could still occur during the first instants of time, once the temperature has cooled down (due to the absence of Joule heating) 17 
and the closest oxygen ions have drifted away, oxygen diffusion is expected to be the main process governing the resistance change. From a physical perspective it is generally assumed that the slowest process limiting the speed of redox-based resistive switching devices is the ion hopping, as there are many hops involved to travel a certain distance, compared to other processes such as redox reactions which only involve a single transition at an interface. ${ }^{[30]}$ By extrapolating the high temperature diffusion coefficients measured by Burriel et al. in epitaxial thin films ${ }^{[9]}$, the characteristic diffusion length for L2NO4 at room temperature can be estimated, giving a value of $635.3 \mathrm{~nm}$ along the $a b$ plane and of $0.86 \mathrm{~nm}$ along the $c$ axis for at time of $100 \mathrm{~s}$. This Fickian diffusion process would lower the doping concentration, increasing the depletion width thus also $\mathrm{R}_{\mathrm{C}, \mathrm{Ti}}$ and the total resistance of the device. This effect is more important for the oxygen-annealed sample as a higher gradient of interstitial oxygen ions has to re-equilibrate. The orientation of the film crystal grains is expected to play an important role in the rate of the oxygen drift and diffusion processes (faster along the $a b$ plane). However, to univocally assess the orientation effect on the memristive response of Ti/L2NO4/Pt devices, these would have to be built using La2NO4 single crystals such as the ones used by Burriel et al. ${ }^{[12]}$ to measure the diffusion coefficients (at high temperatures) along the two perpendicular crystallographic directions of interest.

The oxygen-annealed sample stands out in the relative resistance change it experienced during and after initialization. This sample shows a more than a 20 -fold increase in resistance while the argon and hydrogen-annealed samples only show an $82 \%$ and $34 \%$ increase, respectively. The larger resistivity of the reduced L2NO4 samples leads to a large voltage drop across the film. Conversely, this voltage drop will be concentrated mostly at the Ti/L2NO4 junction in the case of the more conducting oxygen-annealed sample, especially in the presence of a continuous $\mathrm{TiO}_{\mathrm{x}}$ interlayer. The formation of a thicker and/or more oxidized (thus more resistive) $\mathrm{TiO}_{\mathrm{x}}$ interlayer in this sample due to the field-induced drift of oxygen from L2NO4 18 
into the Ti electrode is expected to result in a large relative resistance increase. On the other hand, the lower amount of oxygen ions available in L2NO4 combined with a smaller electric field at the Ti/L2NO4 junction and a higher IRS value results in a smaller relative resistance change for the reduced samples, possibly related to a smaller oxygen ion drift and more difficult oxidation of the Ti electrode. This then explains the smaller programming window measured for the reduced samples.
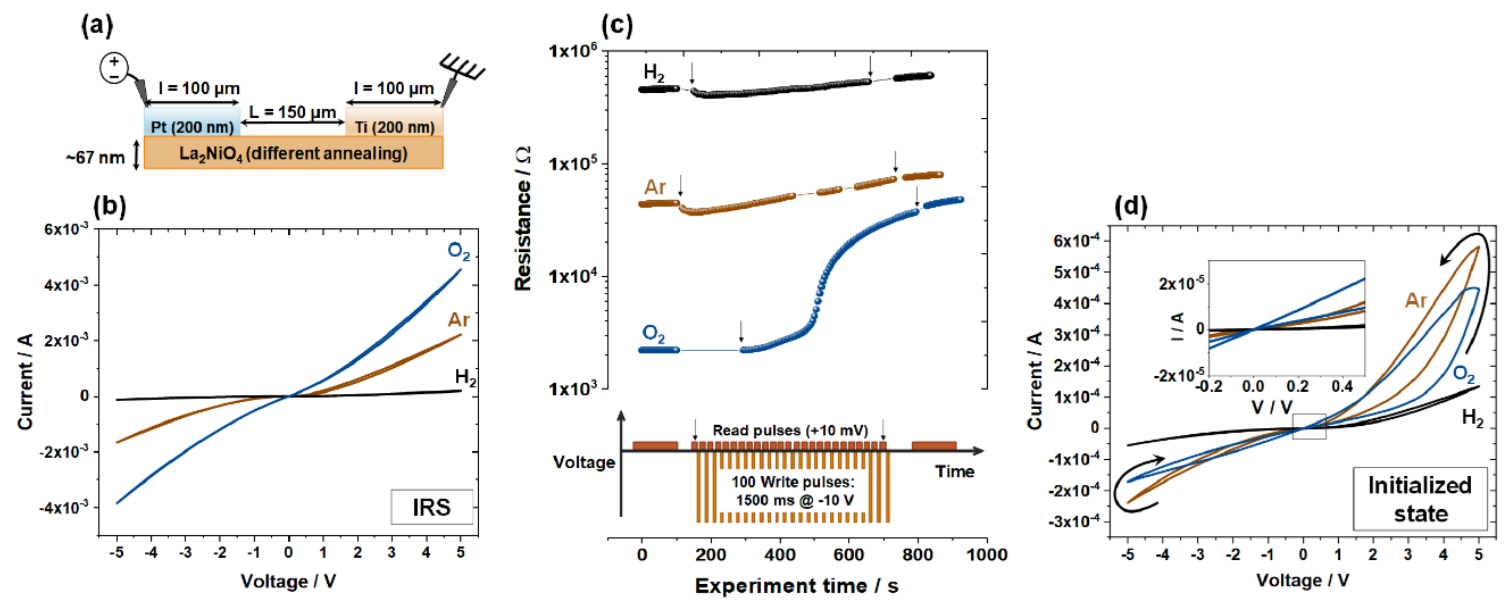

Figure 5: (a) Sketch of the sample showing the biasing conditions. (b) I-V characteristics of the Pt/L2NO4/Ti devices annealed in $\mathrm{O}_{2}$, Ar or $\mathrm{H}_{2} / \mathrm{Ar}$ in their IRS. (c) Initialization procedure of the three devices. Only the read pulses are shown, the vertical black arrows show the start and the end of the write sequence (every fifth read pulse is shown to increase readability). An illustration of the programming sequence is shown at the bottom of the figure. (d) I-V characteristics of the three devices after initialization. The inset is a magnification of the $\mathrm{I}-\mathrm{V}$ curve between $-0.2 \mathrm{~V}$ and $+0.5 \mathrm{~V}$.

The I-V characteristics of the devices in their IRS are displayed in Figure 5.b in which a nonlinear behavior with little rectification for the two reduced samples and almost no rectification for the oxidized sample can be observed. The absence of rectification can be an important marker of an inhomogeneous Schottky barrier height. ${ }^{[31]}$ The I-V characteristics of the initialized samples are shown in Figure 5.d shows hysteretic memristive behavior with very gradual transitions both for the SET and RESET operations. The inset (corresponding to a 
magnification of the electrical characteristics at low voltage) puts in evidence that only the oxygen-annealed sample has a significant difference in the HRS and LRS when cycling the devices between +5 and $-5 \mathrm{~V}$. The presence of two well differentiated resistance states at low voltages (close to $0 \mathrm{~V}$ ) is a signature of better memory retention capabilities for this device. The bipolar characteristics (using write and erase pulses) as well as the relaxation behavior of the L2NO4-based memory were further analyzed for the optimized oxygen-annealed devices for which multilevel, analog-type memory programing capabilities have been demonstrated (see details in the SI, Figure S5).

Under the $\pm 5 \mathrm{~V}$ biasing conditions used (see Figure 5.d) only the oxygen-annealed sample has memory retention, while the changes induced in the reduced samples are volatile. The argon-annealed sample has a hysteretic behavior for $\mathrm{V}>300 \mathrm{mV}$, however the opening of the IV curve disappears at low voltages, while the hydrogen-annealed sample has a very small opening for $\mathrm{V}>1 \mathrm{~V}$. Thus we can conclude that oxygen is clearly playing a major role in the memristive properties of the L2NO4-devices. The oxidation/reduction of $\mathrm{Ti}\left(\mathrm{TiO}_{\mathrm{x}}\right)$ could explain the varying retention characteristics. While redox reactions are likely present in the oxygen-annealed case, in the reduced samples the hysteresis could be due to a temporary variation in the hole-doping concentration at the Ti/L2NO4 interface, changing its electrical characteristics in a reversible and volatile manner.

It can thus be concluded that for the future scaling-up and integration oxygen-rich L2NO4devices are the most promising ones. The dimensions of the devices could be reduced remaining in a horizontal device structure by advanced lithography techniques or moving to a vertical one using, for example, the epitaxial transfer method on $\mathrm{Si}$ at room temperature. ${ }^{[32]}$ When reducing the dimensions the voltage drop occurring in each of the localized regions of the device should be well controlled to avoid filamentary switching. In addition, a precise interplay between the $\mathrm{TiO}_{\mathrm{x}}$ interlayer thickness, stoichiometry and homogeneity and the 20 
oxygen content of L2NO4 are certainly crucial parameters that can be further optimized to tune the resistance-change and the retention characteristics of the memristive device.

\section{Conclusion}

L2NO4 was selected as mixed ionic-electronic conducting oxide to build memristive devices with tunable oxygen stoichiometry due to its inherent ability to accommodate oxygen interstitials in its structure, due to its fast oxygen diffusion and its semiconducting electronic transport properties. For this highly oriented L2NO4 thin films were first deposited by PiMOCVD on $\mathrm{SrTiO}_{3}$ single crystal substrates and then subjected to different anneal treatments under oxidizing or reducing conditions. The combination of structural (XRD), chemical (XANES), microscopy (SEM and PEEM) and electrical measurement techniques proved the direct relationship between the changes in oxygen content, cell parameter, $\mathrm{Ni}$ oxidation state, work function and resistivity in the L2NO4 films after the anneals. Next, films with three different oxygen stoichiometries were used to build functional memristive devices: platinum was selected as inert ohmic electrode while titanium was selected for its high oxygen affinity, making it a chemically active electrode. As proven by a combination of STEM, EELS maps and VCA analysis titanium formed an amorphous $\mathrm{TiO}_{\mathrm{x}}$ interlayer at the Ti/L2NO4 junction, key for the appearance of memristance. The initialization of the device achieved by applying a train of negative write pulses on the Pt side (in Pt/L2NO4/Ti) results in a resistance increase in all cases. Remarkably the film with the highest oxygen content presents the highest $(2000 \%)$ relative resistance increase and the largest opening of the IV memristance curve after initialization. This study has clearly shown that the additional oxygen ions present in the form of interstitial point defects in L2NO4 give rise to the memristive properties of the device. These promising results offer additional insights into the use of L2NO4-based heterostructures with tuned oxygen content for the development of memristive systems for analog computing and synaptic applications. 


\section{Experimental Section}

Thin film preparation. L2NO4 thin films $(\sim 67 \mathrm{~nm})$ have been deposited on $10 \times 10 \mathrm{~mm}^{2}$ (100) $\mathrm{SrTiO}_{3}$ single crystal substrates purchased from Crystec. The substrates followed a mild 3-step cleaning process (acetone, isopropanol, deionized water) in an ultrasonicating bath before deposition of the L2NO4 film by PiMOCVD (more details about the deposition conditions are given in Table 1).

Post annealing treatments have been used to tune the bulk oxygen content of L2NO4. For this, three different pristine (as deposited) L2NO4/STO samples have been annealed in oxidizing $\left(\mathrm{O}_{2}\right)$ or reducing $\left(\mathrm{Ar}\right.$ and $\mathrm{H}_{2}(6 \%) / \mathrm{Ar}$ ) atmospheres at $500{ }^{\circ} \mathrm{C}$ during $1 \mathrm{~h}$, with a heating ramp of $10{ }^{\circ} \mathrm{C} / \mathrm{min}$ and natural convection to cool down the sample back to room temperature.

Metallic electrodes have been fabricated in high vacuum conditions by e-beam induced metal evaporation (MEB550 from PLASSYS) using a laser-assisted lithography process ( $\mu$ PG 101 from HEIDELBERG Instruments) in local cleanroom facilities. A mild $\mathrm{Ar}^{+}$etch has been used to prepare the exposed surface of the patterned sample and remove potential left-overs of the photoresist before metal evaporation. $200 \mathrm{~nm}$ thick $\mathrm{Pt}$ and $\mathrm{Ti}$ electrodes have been deposited (with a growth rate of $0.5 \mathrm{~nm} / \mathrm{s}$ ) to provide sufficient mechanical resistance and prevent potential degradation during electrical contacting.

Phase identification, structural and crystallinity characterization of the samples have been performed using X-ray diffraction (XRD) in $\theta-2 \theta$ geometry (Bruker D8 Advance) with monochromatic $\mathrm{CuK}_{\alpha 1}$ radiation $(\lambda=1.5406 \AA)$.

The resistivity of the films has been measured using a 4-probe setup.

XANES experiments have been carried out on the CRG beamline SpLine (BM25A) at the European Synchrotron Radiation Facility (ESRF), more details about the experiment are available in the SI. 
PEEM measurements were carried out with a NanoESCA MkI spectromicroscope (ScientaOmicron) in ultra-high vacuum (base pressure $2 \times 10^{-10} \mathrm{mbar}$ ) on samples treated beforehand using argon cluster ions in order to remove the adventitious carbon layer without damaging the oxide material underneath. VUV excitation (He I, hv=21.22 eV) and aberrationcompensated imaging spectrometer provided secondary electron spectra from which the work function threshold was determined within $\pm 100 \mathrm{meV}$. The energy scale of the spectra was referenced to the Fermi level of the sample.

The lamellas for the STEM experiments have been prepared by Focused Ion Beam (FIB) using a FEI strata $400 \mathrm{~S}$ equipped with a dual beam electron/ $\mathrm{Ga}^{+}$ions. The region of interest (ROI) is thinned to approximately $100 \mathrm{~nm}$ using a decreasing acceleration, from $16 \mathrm{kV}$ to 2 $\mathrm{kV}$ in order to limit the amorphization of the sample. The high resolution STEM images have been acquired on a FEI TITAN Themis microscope with an electron acceleration of $200 \mathrm{kV}$. The microscope is probe corrected.

EELS composition maps have been obtained using a conventional analysis with the "Gatan GMS 3.20", integrating the signal under the respective element edges: O-K ( 532eV), Ti-L2 $(\sim 462 \mathrm{eV})$ and L3 $(\sim 456 \mathrm{eV}), \mathrm{Ni}-\mathrm{L} 2(\sim 872 \mathrm{eV})$ and L3( 855eV) and La-M4 $(\sim 849 \mathrm{eV})$ and M5( 832eV) (taking in account the plural scattering).

VCA analysis: An advanced hyperspectral unmixing method was used to observe the variations in the environment of the $\mathrm{O}$ K-edge spectrum acquired by EELS by looking at its fine structure. The unmixing of the main end-member components was carried out using the Vertex Component Analysis (VCA) algorithm developed by J.M.P Nascimento and J.M. Bioucas-Dias. ${ }^{[33]}$ The use of a VCA algorithm constitutes a highly relevant method for discriminating EELS fine structures in high resolution hyperspectral images. ${ }^{[34]}$ The automated determination of the spectral components limits the operator-induced variability and helps determining more precisely the regions which each of the components is present or 23 
where a mixture of components co-exist. Further constrains in the automatically generated EELS maps ensure that the results remain physically meaningful. Four components were required for the spectral unmixing to properly define all the pure end-members components present in a consistent and reproducible manner for all the EELS maps regions measured. A spectral unmixing using five components was also attempted but the fifth component generated undesired artefacts (mainly in the form of peak compensation in the background component). More details on the VCA data treatment are given in SI.

Current-Voltage (I-V) characteristics have been obtained using a source-measurement unit (Agilent B1500) using two tungsten-carbide probes operated in a voltage-control mode in a Karl-Suss probe station (acting as a vibration damper, a Faraday cage and a dark box to limit outside disturbances). The voltage is always applied on the Pt electrode while the Ti counter electrode remains grounded. The bipolar sweeps always start at zero bias and go as follows: 0 $\mathrm{V} \rightarrow+\mathrm{V}_{\max } \rightarrow 0 \mathrm{~V} \rightarrow-\mathrm{V}_{\min } \rightarrow 0 \mathrm{~V}$, with a constant sweeping rate of $\sim 350 \mathrm{mV} / \mathrm{s}$. The resistance values extracted from the $\mathrm{I}-\mathrm{V}$ curves were always calculated at $\mathrm{V}=+10 \mathrm{mV}$. Resistance-time (R-t) characteristics, when operated in pulsed mode, consist in applying a voltage stress at higher voltages $(\geq 1 \mathrm{~V})$ to program the device to a higher or lower resistance state, and reading out the remnant resistance state of the device after each programing pulse at a lower $\mathrm{V}=+10 \mathrm{mV}$ bias to ensure that no ionic movement would be triggered by the electrical field. The standardized initialization procedure (illustrated at the bottom of Figure 5.c) consists of the initial readout of the IRS at $+10 \mathrm{mV}$ during $100 \mathrm{~s}$ to test its stability over time, followed by a train of 100 write/read $([-10 \mathrm{~V} ; 1500 \mathrm{~ms}] /[+10 \mathrm{mV} ; 250 \mathrm{~ms}])$ pulses and, finally, another $100 \mathrm{~s}$ long readout at $+10 \mathrm{mV}$ to probe the new resistance state of and measure its stability over time. All the electrical measurements have been carried out in ambient conditions.

\section{Supporting Information}


Supporting Information is available from the Wiley Online Library or from the author.

\section{Acknowledgements}

This work has been partially supported by the LabEx Minos ANR-10-LABX-55-01 and by two ANR funded projects MICROSWITCH (ANR-14-ACHN-0012) and Alps Memories (ANR-15-CE24-0018). We acknowledge the European Synchrotron Radiation Facility (ESRF), the Spanish Ministry of Science, Innovation and Universities (MCIU), and The Spanish National Research Council (CSIC) for provision of synchrotron radiation facilities and we would like to thank beamline staff for assistance in using beamline CRG BM25SpLine. E.S.-C. and G.R.C. thank the MCIU and the CSIC for financially supporting this research project and the beamline operation under Grant No. PIE 2010-6-OE-013.

K. Momma and F. Izumi are kindly acknowledged for creating the VESTA ${ }^{[35]}$ software (3D visualization of crystallographic structure).

The authors declare no competing financial interest. 


\section{References}

[1] R. Waser, J. Nanosci. Nanotechnol. 2012, 12, 7628.

[2] N. K. Upadhyay, H. Jiang, Z. Wang, S. Asapu, Q. Xia, J. Joshua Yang, Adv. Mater. Technol. 2019, 4, 1 .

[3] S. Bagdzevicius, K. Maas, M. Boudard, M. Burriel, J. Electroceramics 2017, 39, 157.

[4] M. Burriel, H. Téllez, R. J. Chater, R. Castaing, P. Veber, M. Zaghrioui, T. Ishihara, J. A. Kilner, J.-M. Bassat, J. Phys. Chem. C 2016, 120, 17927.

[5] J. M. Tranquada, Y. Kong, J. E. Lorenzo, D. J. Buttrey, D. E. Rice, V. Sachan, Phys. Rev. B 1994, 50, 6340 .

[6] A. Chroneos, D. Parfitt, J. A. Kilner, R. W. Grimes, J. Mater. Chem. 2010, 20, 266.

[7] S. J. Skinner, Solid State Sci. 2003, 5, 419.

[8] J. Bassat, Solid State Ionics 2004, 167, 341.

[9] M. Burriel, G. Garcia, J. Santiso, J. A. Kilner, R. J. Chater, S. J. Skinner, J. Mater. Chem. 2008, 18, 416.

[10] A. Chroneos, R. V. Vovk, I. L. Goulatis, L. I. Goulatis, J. Alloys Compd. 2010, 494, 190.

[11] L. Minervini, R. W. Grimes, J. A. Kilner, K. E. Sickafus, J. Mater. Chem. 2000, 10, 2349.

[12] M. Burriel, H. Téllez, R. J. Chater, R. Castaing, P. Veber, M. Zaghrioui, T. Ishihara, J. A. Kilner, J.-M. Bassat, J. Phys. Chem. C 2016, 120, 17927.

[13] C. Baeumer, C. Schmitz, A. H. H. Ramadan, H. Du, K. Skaja, V. Feyer, P. Müller, B. Arndt, C.-L. Jia, J. Mayer, R. a. De Souza, C. Michael Schneider, R. Waser, R. Dittmann, S. Figure, Nat. Commun. 2015, 6, 8610.

[14] K. Maas, E. Villepreux, D. Cooper, C. Jiménez, H. Roussel, L. Rapenne, X. Mescot, Q. Rafhay, M. Boudard, M. Burriel, J. Mater. Chem. C 2020, 8, 464. 
[15] P. L. Bach, J. M. Vila-Fungueiriño, V. Leborán, E. Ferreiro-Vila, B. RodríguezGonzález, F. Rivadulla, APL Mater. 2013, 1, DOI 10.1063/1.4818356.

[16] J. M. Bassat, P. Odier, J. P. Loup, J. Solid State Chem. 1994, 110, 124.

[17] J. B. Goodenough, S. Ramasesha, Mater. Res. Bull. 1982, 17, 383.

[18] T. D. Thanh, L. V. V Bau, N. M. Dang, L. V. V Hong, S.-C. Yu, H. T. Van, D. T. A. Thu, L. V. V Bau, D. N. H. Nam, N. M. An, L. V. V Hong, S.-C. Yu, IEEE Trans. Magn. 2017, 9464, 1.

[19] M. H. Jang, R. Agarwal, P. Nukala, D. Choi, A. T. C. Johnson, I. W. Chen, R. Agarwal, Nano Lett. 2016, 16, 2139.

[20] R. J. Woolley, B. N. Illy, M. P. Ryan, S. J. Skinner, J. Mater. Chem. 2011, 21, 18592.

[21] M. Zinkevich, F. Aldinger, J. Alloys Compd. 2004, 375, 147.

[22] W. R. Acevedo, C. Ferreyra, M. J. Sánchez, C. Acha, R. Gay, D. Rubi, J. Phys. D. Appl. Phys. 2018, 51, DOI 10.1088/1361-6463/aaaed6.

[23] A. Herpers, C. Lenser, C. Park, F. Offi, F. Borgatti, G. Panaccione, S. Menzel, R. Waser, R. Dittmann, Adv. Mater. 2014, 26, 2730.

[24] S. Asanuma, H. Akoh, H. Yamada, A. Sawa, Phys. Rev. B 2009, 80, 235113.

[25] K. Shono, H. Kawano, T. Yokota, M. Gomi, Appl. Phys. Express 2008, 1, 0550021.

[26] D. A. Muller, N. Nakagawa, A. Ohtomo, J. L. Grazul, H. Y. Hwang, Nature 2004, 430, 657.

[27] O. Ravkina, J. Räthel, A. Feldhoff, J. Eur. Ceram. Soc. 2015, 35, 2833.

[28] G. Bertoni, E. Beyers, J. Verbeeck, M. Mertens, P. Cool, E. F. Vansant, G. Van Tendeloo, Ultramicroscopy 2006, 106, 630.

[29] M. Sayer, P. Odier, J. Solid State Chem. 1987, 67, 26.

[30] S. Menzel, M. von Witzleben, V. Havel, U. Böttger, Faraday Discuss. 2018, DOI 10.1039/c8fd00117k. 
[31] R. T. Tung, Appl. Phys. Rev. 2014, 1, 011304.

[32] S. R. Bakaul, C. R. Serrao, M. Lee, C. W. Yeung, A. Sarker, S.-L. Hsu, A. K. Yadav, L. Dedon, L. You, A. I. Khan, J. D. Clarkson, C. Hu, R. Ramesh, S. Salahuddin, Nat. Commun. 2016, 7, 10547.

[33] J. M. P. Nascimento, J. M. B. Dias, IEEE Trans. Geosci. Remote Sens. 2005, 43, 898.

[34] N. Dobigeon, N. Brun, Ultramicroscopy 2012, 120, 25.

[35] K. Momma, F. Izumi, J. Appl. Crystallogr. 2011, 44, 1272. 